\title{
L'AUTEUR EST MORT, VIVENT LES PERSONNAGES ! LA NOTION DE LIBERTÉ À TRAVERS LES JEUX NARRATIFS DE LA SIMULATION ET DE LA DISSIMULATION DANS LA SEPTIÈME FONCTION DU LANGAGE DE LAURENT BINET
}

\author{
Petr VURM
}

Université Masaryk de Brno

\begin{abstract}
En): The paper deals with the simulation and dissimulation of reality, as well as the consequences of the death of the author suggested by Roland Barthes, in the novel $L a$ septième fonction du langage by Laurent Binet. The novel itself doesn't fit any precise subgeneric category, standing on the border between a detective and/or spy novel, a semiological quest and a parody of the intelligentsia of the French theory. Through our analyses, we purport to demonstrate that the author/narrator can (dis)simulate a liberation of the main character from his own "tutelage". The theory of simulated reality is also approached towards the end of the paper.
\end{abstract}

Keywords (En): Laurent Binet ; La septième fonction du langage ; Roland Barthes ; simulation ; dissimulation ; death of the author ; freedom ; literary character ; narrative games ; simulated reality

Mots-clés (Fr): Laurent Binet ; La septième fonction du langage ; Roland Barthes ; simulation ; dissimulation ; mort de l'auteur ; personnage littéraire ; jeux narratifs ; réalité simulée

\section{Introduction}

Le livre La septième fonction du langage de Laurent Binet ne cesse d'attirer, depuis sa publication, l'attention de nombreux critiques littéraires. Cela témoigne de plusieurs choses : Laurent Binet, grâce à son récit savamment construit, nous invite à participer à l'enquête policière concernant la mort de Roland Barthes. Ce faisant, il nous amène à réfléchir sur la littérature et sur les théories littéraires, ainsi que sur le langage. En plus, et au-delà du précédent, à travers le roman faussement policier, l'auteur touche les questions fondamentales, voire philosophiques, de toute écriture et de toute littérature. Est-ce là que se cache le succès de ce roman? Permettons-nous une question d'ordre générique d'abord: que sommes-nous en train de lire? Est-ce un roman à mystère, un polar intellectuel, polar pour intellectuels, polar pour connaisseurs des années 19601980 en France et de la French theory, ou bien s'agit-il d'une farce intellectuelle, d'une énigme complexe voire d'une série d'interprétation sémiologique des signes qui abondent dans le récit ? En effet, l'œuvre analysée s'offre à des lectures multiples, sans qu'on puisse déterminer qu'une seule parmi celles-ci soit la lecture juste : ainsi, le lecteur peut suivre l'enquête policière, s'amuser à reconnaittre les grands personnages intellectuels, prendre le plaisir de les voir dans des situations désobligeantes ou même il peut s'identifier avec les personnages principaux et se délecter à déchiffrer les signes et les symboles à la façon d'un sémiologue acharné.

Avant et surtout, le lecteur peut s'amuser à dévoiler une satire de l'intelligentsia divine ou divinisée de la France, un plaisir comparable à celui qu'a 
dû éprouver Laurent Binet en écrivant son texte. Egalement, à l'époque de la postvérité et des faits alternatifs, nous pouvons, en tant que lecteurs, nous réjouir, grâce à cette fiction, à voir se dérouler sous nos yeux la simulation d'une des réalités possibles, plus encore, nous pouvons jouer le jeu consistant à remplir les interstices de cette «machine paresseuse qui exige du lecteur un travail coopératif acharné pour remplir les espaces de non-dit ou de déjà-dit restés en blanc » (ECO, 1985 : 29). Autrement dit, même si ce n'est pas vrai, cela aurait pu être vrai, et d'ailleurs, qu'est-ce que la vérité et où se cache-t-elle ? Car les petits et les grands détails de l'Histoire (et de l'histoire d'ailleurs) nous échapperont pour toujours. Or, avec Laurent Binet, nous pouvons lire au sujet de la mort réelle de Roland Barthes, écrasé à Paris le 25 février 1980 par une camionnette, un fait historique réel, simulé et dissimulé par le biais de la fiction : nous pouvons alors suivre la spéculation centrale du récit qu'il s'agit d'un assassinat ourdi par la maffia bulgare. D'un assassinat politique de surcroît ! À la veille de l'élection présidentielle de 1981, la fonction magique du langage constitue un objet convoité par les politiciens tels que Valéry Giscard d'Estaing et François Mitterrand, secondés de leurs acolytes, mais aussi par les plus brillants intellectuels français du temps. Nous voyons les personnalités, et avouons-le, chacun parmi nous a sa bete noire, D'Althusser à Lacan, de Foucault à Derrida, via Sollers, Kristeva, Cixous et Bernard Henri-Lévy, de Deleuze à Baudrillard, qui deviennent des personnages de fiction souvent loufoques et comiques d'un polar sémiologique comique voire grotesque, présentés avec une distance plus ou moins ironique.

Qu'est-ce que la sémiologie ? s'interroge justement Eco, à propos de son ami sémiologue écrasé. Un couple bizarre d'enquêteurs - un policier un tantinet grossier mais brave comme l'a été son homonyme Bayard, assisté de Simon Herzog, un jeune intellectuel choisi à la fac de Vincennes - vient interroger le célèbre sémiologue à Bologne parce que son nom a été prononcé par un mystérieux témoin. Comme on l'explique à l'inspecteur chargé de l'enquête, qui malgré son nom héroïque ou exactement à cause de cela n'a absolument aucune idée de ce qu'est la sémiologie, "C'est apprendre à voir le monde, dans sa globalité, comme un ensemble de faits signifiants. » (BINET, 2015 ${ }^{1}$ : 219)

Les personnages s'engagent à faire du langage une arme contre le fondement d'un pouvoir politique. Telle est, selon la fiction simulée par le roman, la dernière découverte du linguiste russo-américain Roman Jakobson, maître de Barthes, toujours vivant en 1980 et théoricien des six fonctions du langage. La présumée septième, magique, pourvoyant accès à tous les pouvoirs liés à la parole - aurait été en possession de Roland Barthes. Ce qui entraine la mort du célèbre auteur, en attisant les appétits des politiciens et intellectuels français, avides de s'emparer du texte «sacré ». Insolente, facétieuse et drôle, cette satire d'une intelligentsia narcissique avide de pouvoir fait rire son lecteur. Mais au-delà du rire, il s'agit d'une fine déconstruction du romanesque, des mots et surtout de la langue et du langage. Dans notre monde actuel qui hésite de plus en plus entre la vérité et le mensonge, comment distinguer le réel de la fiction dans nos sociétés, comment comprendre si l'on se trouve dans une simulation qui serait en même temps une

\footnotetext{
${ }^{1}$ BINET, 2015 raccourci $« B$ » dans la suite.
} 
dissimulation de la réalité ? À quels signes se fier ? Et, en effet, pourquoi ?

\section{La question de la question}

Or, nous constatons que La septième fonction du langage est un roman où, sous les apparences d'un roman d'aventures et d'un polar intellectuel, est dissimulée toute une quête sémiologique complexe - une chaîne interprétative de signes qui sont reliés l'un à l'autre à travers l'histoire : un signe interprété devient ensuite un signe à interpréter et ainsi de suite. La stratégie de simulation et de dissimulation dans le roman est alors un jeu de pistes complexes, dont de beaucoup ne mènent nulle part, servant à confondre à la fois les adversaires, les personnages et le lecteur. L'exemple emblématique de cette confusion entre le vrai et le faux étant le faux document écrit de la présumée septième fonction, qui, à la différence du texte original, ne fonctionne pas.

Pour compléter les investigations théoriques effectuées dans ce même recueil par Petr Kyloušek et Veronika Černíková, portant sur le même roman, nous nous sommes inspiré de la fin du livre où Simon Herzog mène une lutte à la fois contre la maffia italienne et, en parallèle, contre son auteur. Or, dans le suivant, nous aborderons la problématique liée à la notion barthésienne de la mort de l'auteur, celle portant sur la relation entre la simulation et la dissimulation du rapport entre la personne réelle et le personnage, celle de la relation entre l'intention et l'interprétation, et finalement celle de la notion spéculative de la liberté du personnage vis-à-vis de son auteur. Pour comprendre les enjeux complexes de la stratégie narrative binetienne, nous devrions nous poser des questions fines, dignes de la plume de l'auteur de ce livre. L'une des difficultés de la stratégie sophistiquée consistant en un jeu dynamique entre la simulation et la dissimulation repose sur le fait que les protagonistes, tels qu'ils nous sont présentés dans le roman, ne sont évidemment pas des personnes réelles, même s'ils portent de nombreuses caractéristiques telles qu'on les connaît de l'histoire de la philosophie et de la critique littéraire. Mais ils ne sont pas des personnages de fiction classiques non plus parce qu'ils sont en même temps les énonciateurs des théories littéraires et linguistiques qui gèrent la narration, dépassant ainsi le cadre de la narration classique. Dans cette fiction complexe, qui représente un imbroglio de signes à déchiffrer, il est assez difficile d'identifier d'emblée et assez clairement les fonctions narratives exactes et les rôles attribués aux protagonistes.

Or, la première question-clé que nous devons nous poser n'est sans doute pas celle du sous-titre du roman, à savoir Qui a tué Roland Barthes, ce qui est déjà une des premières dissimulations de la signification véritable de ce roman. Il serait trop facile de tomber dans le piège de la simulation d'un roman policier dès la couverture du roman. La première question à nous poser est donc : Qui est Roland Barthes. Alors posons-nous cette question : qui est Roland Barthes critique et qui est Roland Barthes personnage ?

Roland Barthes, né le 12 novembre 1915 à Cherbourg et mort le 26 mars 1980 à Paris, est un philosophe, critique littéraire et sémiologue français. Roland Barthes est, entre autres, l'auteur de Mythologies, du Degré zéro de l'écriture et de l'Empire des signes. Egalement, Barthes semble avoir signé un article qui, en 
1968, a fait couler beaucoup d'encre : il semblerait ainsi être l'auteur d'un article intitulé La mort de l'auteur. Sauf que dans le même article, Barthes constate la mort de l'auteur au profit de l'interprétation effectuée par le lecteur, et donc il serait fort irrévérencieux de notre part de l'appeler un auteur contre son gré. Cependant, il ne serait pas juste non plus de dire que Roland Barthes n'a rien à voir avec l'article intitulé La mort de l'auteur. Disons alors tout simplement que Roland Barthes peut être vaguement associé à cet article et que ses activités intellectuelles de 1968 coïncident de manière fort surprenante avec la publication dudit article. Il y aurait donc une relation de cause à effet entre Roland Barthes et l'existence de l'article La mort de l'auteur en question.

\section{La mort de l'auteur de La mort de l'auteur}

Expliquons brièvement, pour le propos de notre argument, l'idée principale de cet article célèbre, qui est, espérons-le, généralement connue. À l'auteur comme principe producteur et explicateur de la littérature, Barthes substitue le langage, impersonnel et anonyme, peu à peu revendiqué comme matière exclusive de la littérature par Mallarmé, Valéry, Proust, le surréalisme, ou encore : «L'écriture, c'est ce neutre, ce composite, cet oblique où fuit notre sujet, le noir-et-blanc où vient se perdre toute identité, à commencer par celle-là même du corps qui écrit » (BARTHES, 1968 : 61). Pour Barthes, « c'est le langage qui parle, ce n'est pas l'auteur » (Ibid). L'auteur ainsi disqualifié, le seul sujet en question dans la littérature est celui de l'énonciation : «l'auteur n'est jamais rien de plus que celui qui écrit, tout comme je n'est autre que celui qui dit je » (BARTHES, $1968: 63$ ). L'auteur cède donc le devant de la scène à l'écriture, au texte, ou encore au scripteur, qui n'est jamais qu'un « sujet » au sens grammatical ou linguistique, un être de papier, non une «personne » au sens psychologique : c'est le sujet de l'énonciation, qui ne préexiste pas à son énonciation mais se produit avec elle, ici et maintenant. L'auteur n'est rien de plus qu'un copiste mêlant les écritures, loin de tout mythe de l'origine et de l'originalité. D'où il s'ensuit encore que l'écriture ne peut pas «représenter», «peindre » quoi que ce soit qui serait préalable à son énonciation, et qu'elle n'a pas plus d'origine que n'en a le langage. Quant à l'explication, elle disparaît avec l'auteur, puisqu'il n'y a pas de sens unique, originel, au principe, au fond du texte. Bref, la critique doit faire l'impasse sur l'auteur: " Donner un Auteur à un texte, c'est imposer à ce texte un cran d'arrêt, c'est le pourvoir d'un signifié dernier, c'est fermer l'écriture» (BARTHES, 1968 : 68).

La lecture ne correspond pas à un déchiffrement critique, mais à une appropriation : «La naissance du lecteur doit se payer de la mort de l'Auteur » (BARTHES, 1968 : 69), comme obstacle à la liberté de la lecture. Ainsi, dernier maillon du nouveau système qui se déduit en entier de la mort de l'auteur : le lecteur, et non l'auteur, est le lieu où l'unité du texte se produit, dans sa destination au lieu de son origine, mais ce lecteur n'est pas plus personnel que l'auteur tout juste déboulonné, et il s'identifie lui aussi à une fonction : il est «ce quelqu'un qui tient rassemblées dans un même champ toutes les traces dont est constitué l'écrit » (BARTHES, 1968 : 67). L'extrait suivant résume l'idée principale : 
Nous savons maintenant qu'un texte n'est pas fait d'une ligne de mots, dégageant un sens unique, en quelque sorte théologique (qui serait le «message » de l'Auteur-Dieu), mais un espace à dimensions multiples, où se marient et se contestent des écritures variées, dont aucune n'est originelle : le texte est un tissu de citations, issues des mille foyers de la culture. Pareil à Bouvard et Pécuchet, ces éternels copistes, à la fois sublimes et comiques, et dont le profond ridicule désigne précisément la vérité de l'écriture, l'écrivain ne peut qu'imiter un geste toujours antérieur, jamais originel ; son seul pouvoir est de mêler les écritures, de les contrarier les unes par les autres, de façon à ne jamais prendre appui sur l'une d'elles ; (BARTHES, 1968 : 65).

Pour compléter cette présentation par un autre point de vue, reprenons les mots d'Antoine Compagnon, de son cours intitulé - Introduction : mort et résurrection de l'auteur:

Le conflit peut encore être décrit comme celui des partisans de l'explication littéraire, comme recherche de l'intention de l'auteur (on doit chercher dans le texte ce que l'auteur a voulu dire), et des adeptes de l'interprétation littéraire, comme description des significations de l'œuvre (on doit chercher dans le texte ce qu'il dit, indépendamment des intentions de son auteur). Pour échapper à cette alternative, une troisième voie, souvent privilégiée aujourd'hui, insiste sur le lecteur comme critère de la signification littéraire. (COMPAGNON, 2008)

Comme nous l'avons déjà indiqué au début, toutes les traces du récit gravitent autour d'un seul concept qui est celui du langage. Le langage n'est pas qu'un outil de communication, mais il est surtout, grâce à son pouvoir rhétorique, conatif et incantatoire, l'arme de combat de l'autorité et la source du pouvoir symbolique dans le sens de Pierre BoURDIEU (2001). La fonction du langage participe alors à une tension dynamique constante entre le savoir et le pouvoir. La démythisation qu'apporte Binet au récit consiste dans le fait qu'il démontre, entre autres, que les grands intellectuels du récit participent à toutes sortes de simulations intellectuelles liées au savoir, pour dissimuler, au fond, leur volonté véritable et fondamentale qui est celle d'obtenir le pouvoir d'abord symbolique, ensuite politique et réel. Leur mission va au rebours du décentrement opéré par Barthes, c'est-à-dire, dans la dichotomie de l'intention et de l'interprétation, ils manifestent une intention d'autorité sur leurs co-personnages en aspirant à pouvoir persuader et contrôler leur audience.

\section{De l'auteur mort aux personnages morts et vivants}

Pour résumer ce qui précède et pour revenir à Roland Barthes, qui à part être lié à l'article sus-mentionné La mort de l'auteur et qui par l'ironie du sort finit lui aussi comme un auteur mort, il occupe dans la fiction de Laurent Binet une position clé. Il est à la fois la victime du meurtre mais surtout en tant que découvreur et détenteur du secret représenté par la Septième fonction du langage. Cette fonction narrative de Barthes-personnage, comme on l'a vu à propos de son propre texte fondamental, est à la fois littérale et symbolique. Barthes détient littéralement la lettre de la fonction magique, ce qui est la cause réelle de sa mort, mais son article bouleversant est en même temps une clé pour la compréhension du mystère de cette septième fonction inédite, ensemble avec les six fonctions 
précédentes de l'œuvre de Jakobson. Expliquons pourquoi. En effet, la septième fonction, découverte et inscrite par Jakobson, représente un objet de valeur, l'objet magique à la Greimas du récit, convoité par tous. Elle est en quelque sorte la clef de voûte et la fonction de l'interprétation du roman. En même temps, il s'agit d'un texte, qui est, de par sa nature, profondément paradoxal : si Barthes affirme que la naissance du lecteur doit se payer de la mort de l'auteur et que c'est le seul lecteur qui doit remplir les vides interprétatifs d'un texte, nous pouvons nous demander à juste titre comment la fonction magique du texte pourrait fonctionner et si elle peut réellement fonctionner sur tout lecteur ou auditeur. Le paradoxe intrinsèque consisterait donc dans le fait que le narrateur du livre, pour être capable de séduire son lecteur, devrait être en contrôle du texte qu'il applique à son auditoire. Bien qu'il s'agisse d'une question spéculative, nous croyons que le texte de la septième fonction ne pourrait pas exister à cause de ce paradoxe qui sépare l'intention de l'auteur de l'interprétation du lecteur. Il serait donc magique par le biais d'une simulation/dissimulation double : il ne pourrait pas fonctionner dans notre réalité, cela est bien évident. Mais il nous paraît peu probable qu'il puisse fonctionner selon la logique interne du récit ou celle de Roland Barthes. Si c'était le cas, Barthes deviendrait, in fine, un simulacre de lui-même.

Finalement, après avoir discuté le rôle de Roland Barthes en tant qu' «auteur » et personnage du roman à la fois, observons Simon Herzog, le «personnage principal » et l'une des clés possibles pouvant ouvrir au moins quelques portes principales du roman. Personnage purement fictif du roman, à la différence de bien d'autres, il ne fonctionne pas seulement comme accompagnateur et conseiller de l'inspecteur Bayard, chargé de l'enquête : non, il représente en quelque sorte la fonction interprétative de la chaîne sémiotique posée par le meurtre de Roland Barthes. En tant que tel, il est donc l'Anti-Watson du roman policier, infiniment plus compétent à mener une enquête sémiologique que Bayard, dont le nom chevaleresque est à la fois un retour à la tradition française mais aussi un détournement ironique de la narration historique traditionnelle, et dont la compétence, au contraire, atteint un degré zéro de lecture des signes. Ce qui n'empêche pas le détective Bayard de proférer des remarques désobligeantes, comiques, mais qui très souvent déconstruisent grâce à leur naïveté le discours de la simulation/dissimulation des grands intellectuels. Or, du point de vue de la stratégie narrative établie par Laurent Binet, Herzog est le point focal de la lecture, tourné à la fois vers l'intérieur et vers l'extérieur de la narration, parce qu'il interprète les faits pour l'inspecteur, ce faisant, il fournit des éléments clés au lecteur. Ainsi, Herzog représente le premier lecteur de la narration. Cela se fait souvent avant que se passe la performance réservée au lecteur au sens barthésien, au moins nous ne croyons pas qu'un lecteur empirique puisse effectuer le même travail interprétatif que fait l'étudiant de sémiologie.

Cette position double de Herzog est très intéressante dans le récit, comme il rejoint en quelque sorte ce rôle attribué au lecteur par Barthes qui dit :

Il y a cependant quelqu'un qui entend chaque mot dans sa duplicité, et entend de plus, si l'on peut dire, la surdité même des personnages qui parlent devant lui : ce quelqu'un est précisément le lecteur [...] Ainsi se dévoile l'être total de l'écriture : un texte est fait d'écritures multiples, 
issues de plusieurs cultures et qui entrent les unes avec les autres en dialogue, en parodie, en contestation; mais il y a un lieu où cette multiplicité se rassemble, et ce lieu, ce n'est pas l'auteur, comme on l'a dit jusqu'à présent, c'est le lecteur: le lecteur et l'espace même où s'inscrivent, sans qu'aucune ne se perde, toutes les citations dont est faite une écriture; l'unité d'un texte n'est pas dans son origine, mais dans sa destination, mais cette destination ne peut plus être personnelle : le lecteur est un homme sans histoire, sans biographie, sans psychologie ; il est seulement ce quelqu'un qui tient rassemblés dans un même champ toutes les traces dont est constitué l'écrit. (BARTHES 1968 : 66)

Au fur et à mesure que le récit avance, Simon Herzog devient alors le lecteur par excellence, quoique fictif, se muant en interprète principal et véritablement le seul personnage capable de déchiffrer les signes et de voir au-delà de la simulation/dissimulation, ce qui le hausse au niveau du héros principal dans le sens d'un roman d'aventures classique.

\section{Le personnage et la liberté}

Or, pour en venir à la notion centrale de notre exposé, posons-nous maintenant la question de ce qu'est la liberté au sein de ce récit et de ce qu'est la liberté littéraire, voire celle du personnage du roman. Nous pouvons nous demander en corollaire, à quoi cette liberté s'oppose. Effectivement, il serait intéressant de mesurer le personnage de Simon Herzog à l'aune des grands concepts philosophiques de la liberté et ensuite de ceux qui entravent la liberté comme catégorie philosophique. Cependant, vu les contraintes spatiales de cet article, nous nous limiterons à en choisir une qui est plutôt facile, c'est-à-dire la possibilité d'agir sans contrainte et surtout de s'opposer au déterminisme qui dirige ses actions et qui paraît être aux antipodes de la liberté individuelle.

Le personnage littéraire, à la différence des personnes en chair et en os, semble le moins libre de tous les êtres humains. Il reste toujours à la merci de l'auteur et de son acolyte, le narrateur. A-t-il en fait une possibilité de se libérer de la tutelle auctoriale ? Cela pose bien des questions épineuses. Or, la capacité accrue à interpréter des signes, celle de Simon Herzog dans ce cas concret, mène à une conséquence logique et tout à fait compréhensible. Il serait étonnant qu'un sémiologue compétent, quoique fictif, ne se mette à remettre en cause la consistance logique du système dans lequel il se trouve. Or, Simon Herzog constate, peut-être grâce à une faille dans l'économie narrative causée par l'inattention de l'auteur-narrateur, qu'il n'est peut-être qu'un personnage de roman. Dans le cas de Herzog, qui arrive à Naples dans le dernier chapitre, nous constatons qu'il est désormais un personnage révolté. Cela commence par des symboles :

Simon avait d'abord songé à l'antre de la Sibylle, à Cumes, où personne ne serait venu les chercher, mais il n'a pas retenu l'endroit parce que c'était trop kitsch, trop lourdement symbolique, et les symboles commencent à le fatiguer. Sauf qu'on n'échappe pas si facilement aux symboles... (B : 471)

Dans la suite de l'intrigue, c'est au tour de Simon d'être pris par les mafieux de la construction de Naples. Lorsqu'il est sur le point d'être tué, il abandonne son 
rôle de personnage dans une métalepse narrative :

Simon repense aux Japonais de Venise. Cette fois, il n'y aura pas un deus ex machina pour lui venir en aide? En ses derniers instants, Simon renoue le dialogue avec cette instance transcendante qu'il s'est plu à imaginer: si jamais il était coincé dans un roman, quelle économie narrative nécessiterait qu'il meure à la fin ? Simon recense plusieurs raisons narratologiques, qu'il trouve toutes discutables. Il pense à ce que lui dirait Bayard. « Rappelletoi Tony Curtis dans Les Vikings. » Ouais. Il pense à ce que ferait Jacques, neutraliser l'un des hommes armés, abattre le second avec l'arme du premier, sûrement, mais Bayard n'est pas là et Simon n'est pas Bayard. Simon comprend qu'il n'a rien à attendre d'aucune instance transcendante. Il sent que le romancier, s'il existe, n'est pas son ami. ${ }^{2}$

$[\ldots]$

Non, il n'y aura pas de miracle. Mais, roman ou pas, il ne sera pas dit qu'il se sera laissé faire. Simon ne croit pas au salut, ne croit pas qu'il a une mission sur terre, mais il croit au contraire que rien n'est tout à fait écrit à l'avance et que, quand bien même il serait dans les mains d'un romancier sadique et capricieux, son destin n'est pas encore joué.

Pas encore.

Il faut faire avec ce romancier hypothétique comme avec Dieu : toujours faire comme si Dieu n'existait pas car si Dieu existe, c'est au mieux un mauvais romancier, qui ne mérite ni qu'on le respecte ni qu'on lui obéisse. Il n'est jamais trop tard pour essayer de changer le cours de l'histoire. Si ça se trouve, le romancier imaginaire n'a pas encore pris sa décision. Si ça se trouve, la fin est entre les mains de son personnage, et ce personnage, c'est moi.

Je suis Simon Herzog. Je suis le héros de ma propre histoire. (B : 476)

Finalement, le personnage se méfie de son auteur à tel point qu'il veut cacher ses idées devant lui, parce que le savoir, c'est le pouvoir, rappelons-le une dernière fois :

Il ne veut pas que, si romancier il y a, le romancier comprenne comment il a fait. Il ne sera pas dit que quiconque puisse lire en lui comme dans un livre. (B : 477)

Cette révolte, réelle et symbolique à la fois nous paraît intéressante à plusieurs titres. Premièrement, elle apporte une métalepse quand même assez inattendue, au moins pour un lecteur naïf, au dénouement du récit.

Deuxièmement, elle corrobore notre hypothèse du début de l'article sur la mort de l'auteur. Herzog ne tue pas son auteur par lui-même, ce serait sans doute intéressant, mais il semble le «tuer symboliquement», en lui reniant la capacité suprême, à savoir celle d'imposer son autorité et sa capacité de gérer de façon compétente son univers fictif.

Troisièmement, et cela nous paraît encore plus intéressant, le «romancier hypothétique » est comparé à Dieu, ce qui fait entrer en corrélation la mort de Dieu annoncée par Nietzsche et la mort de l'auteur annoncée par Barthes. Il ne reste qu'à annoncer la mort de l'homme et, heureusement, nous avons Foucault pour faire cela. Dans Les mots et les choses, Michel Foucault reprend l'idée nietzschéenne de la mort de Dieu et l'utilise pour parler de la mort de l'homme :

Plus que la mort de Dieu, ou plutôt dans le sillage de cette mort selon une corrélation profonde

${ }^{2}$ C'est nous qui soulignons. 
avec elle, ce qu'annonce la pensée de Nietzsche, c'est la fin de son meurtrier ; c'est l'éclatement du visage de l'homme dans le rire, et le retour des masques, c'est la dispersion de la profonde coulée du temps par laquelle il se sentait porté et dont il soupçonnait la pression dans l'être même des choses ; c'est l'identité du Retour du Même et de l'absolue dispersion de l'homme. (FouCAULT, $1966: 396-7$ )

Or, le constat est triste. Dieu est mort, l'Auteur est mort, l'homme est mort, sans parler de Barthes : mort à son tour. Ce qui nous paraît également un moment valable pour la critique littéraire, c'est que la mort de l'Auteur posée par la révolte de Simon n'est pas exactement celle annoncée par Roland Barthes où, souvenonsnous, l'auteur était mort pour laisser la place au lecteur. Dans le cas concret du roman en question, c'est un personnage qui se révolte contre son auteur dans un double acte de simulation: faire semblant que le personnage est capable d'une telle révolte, et de dissimulation: on dissimule toutes les preuves logiques qui pourraient révéler que Simon n'est après tout qu'une invention de son Auteur. Bien évidemment, cela signifie également qu'un certain pacte de la simulation doit s'établir entre le narrateur et le lecteur. Désormais, le personnage révolté serait libre d'agir comme il veut, l'auteur-narrateur lui donnant une certaine liberté d'action que le lecteur croirait. Nous croyons à notre tour qu'il s'agit ici d'un acte de simulation/dissimulation formidable à plusieurs titres : l'auteur empirique Laurent Binet, pour jouer un jeu avec son lecteur et pour récompenser son personnage avec lequel il s'identifie peut-être, a joué une partie de simulation pendant laquelle il fait semblant qu'il n'est pas l'autorité suprême du récit. Ainsi, il se dissimule derrière la narration et semble oublier son personnage. Celui-ci en profite pour se révolter et pour tuer symboliquement son Auteur.

Cette implication d'une simulation délibérée, faisant partie de l'économie du récit, paraît comporter plusieurs solutions littéraires ou «techniques », étalées sur plusieurs niveaux. À un premier niveau de lecture, nous pouvons affirmer que toute narration est en fait une simulation d'une des réalités potentielles, qui est en train de s'actualiser et que le personnage, dès sa conception initiale est libre en tant qu'une des fonctions fondamentales de la narration.

À un deuxième niveau, l'auteur-narrateur peut choisir quelque dispositif technique, lié non à sa volonté mais à un hasard quelconque ou à une cause et influence externe : les statistiques, les données scientifiques, les modèles de la probabilité, etc.

À un troisième niveau, l'auteur empirique, vraiment conscient de la nécessité de libérer ses personnages, pourrait anticiper les désirs de ses lecteurs et les laisser décider. Ici encore, le personnage passerait d'une tutelle à l'autre, mais c'est surtout l'existence de ce qu'on appelle fan fiction en anglais, c'est-à-dire les récits créés par des lecteurs mais qui reprennent des personnages bien connus, qui leur accordent le plus de liberté, les laissent vivre le plus librement. Autant d'auteurs, autant de solutions possibles, sans parler des possibilités des nouvelles technologies, où le sort des personnages pourrait être décidé non par l'auteur mais par les lecteurs. 


\section{Et si notre réalité était une simulation?}

En guise de conclusion, nous nous permettons d'évoquer une théorie intéressante concernant la théorie de la simulation, surtout pour notre questionnement sur les liens complexes entre la réalité et sa (dis)simulation. L'hypothèse de simulation stipule que notre réalité observable a pour trame une simulation, similaire à celles effectuées par des ordinateurs, sans que les entités y évoluant puissent la distinguer commodément de la vraie réalité. Cette hypothèse repose elle-même sur le développement de la réalité simulée. On la trouve dès le roman de Daniel Galouye Simulacron-3, mais René Descartes dans ses Méditations métaphysiques, Locke et George Berkeley ont envisagé eux aussi l'hypothèse de mondes de simulacre, dont l'idée se trouve dès le mythe de la Caverne de Platon. L'hypothèse de simulation est devenue sujet de nombreux débats faisant intervenir philosophie et sciences cognitives dans un cadre futurologique, en particulier trans-humaniste. L'hypothèse de simulation se présente au départ comme hypothèse sceptique, voire de conspiration, proposition parmi bien d'autres explorant des réponses sur la nature de la réalité face aux questions philosophiques classiques : Qui sommes-nous, d'où venons-nous, où allons-nous ? Peu de temps après l'an 2000, Nick Bostrom utilise des raisons empiriques pour défendre ce point de vue. Selon ce théoricien, il y a de très fortes chances que des entités telles que nous fassent partie d'une réalité virtuelle ou simulée (Réalité simulée, 2017).

À nous, chers lecteurs et collègues, de réfléchir si nous sommes des personnages d'une simulation quelconque et de nous révolter contre les instigateurs de cette simulation, comme l'a fait Simon Herzog, le héros fictif du roman.

\section{BIBLIOGRAPHIE}

BARTHES Roland (1968), La mort de l'auteur, in: Manteia, no. 5, $4^{e}$ trimestre 1968.

BINET Laurent (2015), La septième fonction du langage, Paris, Grasset.

BOURDIEU Pierre (2001), Langage et pouvoir symbolique, Paris, Seuil.

ECO Umberto (1985), Lector in fabula, Paris, Grasset.

FOUCAULT Michel (1966), Les mots et les Choses (une archéologie des sciences humaines), Paris, Gallimard.

GaLOUYE Daniel F. (1964), Simulacron-3, New York, Bantam.

\section{Webographie}

COMPAGNON Antoine (2008), Introduction: mort et résurrection de l'auteur, disponible comme partie du cours en ligne Théorie de la littérature : qu'est-ce qu'un auteur? sur http://www.fabula.org/compagnon/auteur.php, consulté le 31 mars 2017.

Réalité simulée (2017), Wikipedia, consulté le 3 avril 2017. 